\title{
Region-Based Annotation of Digital Photographs
}

\author{
Claudio Cusano \\ DISCo (Dipartimento di Informatica, Sistemistica e Comunicazione), \\ Università degli Studi di Milano-Bicocca, Viale Sarca 336, 20126 Milano, Italy \\ cusano@disco.unimib.it
}

\begin{abstract}
We propose a region-based method for the annotation of outdoor photographs. First, images are oversegmented using the normalized cut algorithm. Each resulting region is described by color and texture features, and is then classified by a multi-class Support Vector Machine into seven classes: sky, vegetation, snow, water, ground, street, and sand. Finally, a rejection option is applied to discard those regions for which the classifier is not confident enough. For training and evaluation we used more than 12,000 images taken from the LabelMe project.
\end{abstract}

\section{Introduction}

In this paper we propose a region-based method for the annotation of outdoor photographs. Our idea is that the provided annotation could be effectively exploited to improve image processing procedures. By following a content-aware processing approach [3] it could be possible to select the most appropriate image enhancement strategy, or the most appropriate region of interest, by taking into account information about the content of the photographs.

In our proposal, homogeneous regions, obtained by a suitable segmentation algorithm, are assigned to seven different classes, or are labeled as "rejected" when they cannot be reliably assigned to one of the classes considered. The classes considered, sky, vegetation, snow, water, ground, street and sand, could be exploited by content-aware processing strategies.

Our method starts with an over-segmentation of the input images. In order to ensure that regions can be assigned to at most one class, we applied a normalized cut algorithm tuned to select regions composed of highly homogeneous pixels. The regions are then described by low-level features: color distribution is represented by statistical moments and by a color histogram; texture is described by a histogram of rotation invariant local binary patterns. Each region is then independently classified by a multi-class (one vs. one) Support Vector Machine (SVM). Finally, a rejection rule is applied to exclude from the annotation those regions which cannot be reliably assigned to one of the seven classes considered.

We based our experimentation on the LabelMe dataset [17. This dataset is composed of thousands of manually annotated images. We exploited the manual annotation to determine the ground thruth for both the training and the evaluation of the classifier. 


\section{Related Work}

Several approaches have been proposed in the last years for automatic image annotation $20,11,6,7,12,16$. Yuan et al. conducted a study which show how spatial context constraints improve automated image region annotation [23]. They classified the regions segmented by the JSEG method into 11 classes: sky, water, mountain, grass, tree, flower, rock, earth, ground, building, and animal. Boutell et al. presented a framework to handle the cases in which a natural scene can be described by multiple class labels [1]. More in detail, they considered combination of classes obtained from six base classes: beach, sunset, foliage, field, mountain, and urban. Millet et al. proposed a method based on the knowledge of how regions should be spatially arranged [14]. In their approach, the regions are classified individually into the classes sky, water, snow, trees, grass, sand, ground, and buildings, using a Support Vector Machine which returns several hypotheses with associated probabilities. For a more complete review of this topic see the survey by Tsai and Hung [19].

A few works in the literature exploited the annotation of regions to drive image enhancement algorithms. Fredembach et al. observed that some regions have specific colors (memory colors) that are perceived consistently by human observers 8]. Therefore their importance, with respect to color correction, is greater than that of other regions in an image. They proposed a method, based on eigenregions, for the detection of regions of blue sky, vegetation, and skin tone. Similarly, Cooper considered memory colors, from skin, sky, and foliage objects, to improve the prediction the scene illuminant 4]. Gasparini and Schettini proposed a method for color cast removal which exploits color and spatial information to identify regions of probable skin, sky, sea, or vegetation [9]. When this regions cover more than $40 \%$ of the image, the image is classified as having an intrinsic cast, and the cast remover is not applied.

\section{$3 \quad$ Method}

The annotation strategy is composed of four major steps (see Figure 1): i) the input image is segmented into uniform regions; ii) each region is independently described by a set of low-level features; iii) the feature vector is classified by a SVM trained to discriminate between the seven classes considered: sky, vegetation, snow, water, ground, street, and sand; iv) regions corresponding to a low confidence of the classifier are rejected. The following sections provide more details about each step.

\subsection{Segmentation}

To segment the input images we adopted the Normalized Cut framework proposed by Malik et al. [13. Our aim is to split regions which probably contain pixels of multiple classes. Therefore, we limited the algorithm to the initial segmentation step. The algorithm treats image segmentation as a graph partitioning problem and uses a global criterion, the normalized cut, for segmenting the 


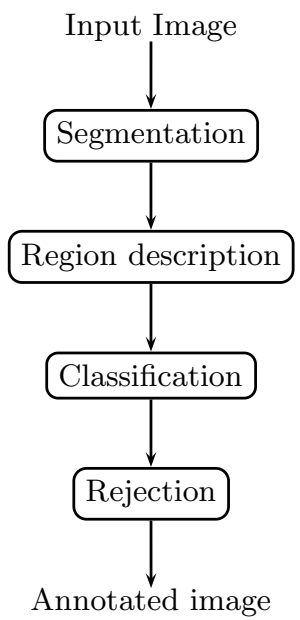

Fig. 1. The main steps which compose the annotation strategy

graph. The normalized cut criterion measures both the total dissimilarity between the different groups as well as the total similarity within the groups.

First, a sparse matrix $\mathbf{W}$ is built to encode the similarity relationships between each pair of pixels in the image. Here, we used a color extension of the weighting scheme proposed by Shi and Malik [18]:

$$
W_{i j}=\left\{\begin{array}{ll}
\exp \left(-\frac{d\left(I_{i}, I_{j}\right)^{2}}{\sigma_{I}^{2}}\right) \exp \left(-\frac{\left\|X_{i}-X_{j}\right\|_{2}^{2}}{\sigma_{X}^{2}}\right) & \text { if }\left\|X_{i}-X_{j}\right\|_{2}<r \\
0 & \text { otherwise }
\end{array},\right.
$$

where $d\left(I_{i}, I_{j}\right)$ is the distance, in the RGB color space, between the values of the $i$-th and the $j$-th pixels in the image; $\left\|X_{i}-X_{j}\right\|_{2}$ is the Euclidean distance between the locations of the two pixels; $\sigma_{I}$ and $\sigma_{X}$ are parameters determining the relative importance of color similarity and spatial proximity; $r$ defines a circular neighborhood for each pixel. In practice, high weights are assigned to pairs of pixels with similar color and close positions.

The normalized cut criterion defines a measure of how well a bipartition of the image keeps similar pixels in the same group:

$$
\operatorname{ncut}(A)=\left(\sum_{i \in A, j \notin A} W_{i j}\right)\left(\frac{1}{\sum_{i \in A, j \in V} W_{i j}}+\frac{1}{\sum_{i \notin A, j \in V} W_{i j}}\right)
$$

where $V$ is the set of all the pixels in the image, which are partitioned into the subsets $A$ and $V \backslash A$.

The problem of minimizing the normalized cut is NP-hard, however an approximate solutions can be found by considering the matrix $\mathbf{W}$ together with the diagonal matrix $\mathbf{D}\left(D_{i i}=\sum_{j} W_{i j}\right)$, and by solving the following generalized eigenproblem:

$$
(\mathbf{D}-\mathbf{W}) \mathbf{y}=\lambda \mathbf{D} \mathbf{y} .
$$


The eigenvectors can be thought of as a transformation of the image into a new feature vector space where the pixels are represented by vectors with the components coming from the corresponding components across the different eigenvectors. The image is segmented by finding the clusters in this eigenvector representation. This is a much simpler problem because the eigenvectors have essentially put regions of coherent descriptors (i.e. connected groups of similar pixels) into tight clusters. We used $K$-means to find an initial subdivision in 30 clusters which is then refined by aggregating those clusters which do not increase too much the quantization error.

\subsection{Regions Description and Classification}

To describe the segmented regions we used three different features describing the texture and the color distribution. The first feature is composed of the first two statistical moments of the components in the YCbCr color space (six components).

The second feature is a 37 bin histogram of the hue channel, inspired by the work of Van de Weijer et al. [21. Since the certainty of the hue is inversely proportional to the saturation, the hue histogram is made more robust by weighting each sample of the hue by its saturation. More in detail, given a region of $N$ pixels, the bins $B_{1}, \ldots B_{37}$ of the histogram are populated as follow:

$$
\begin{aligned}
B_{k} & =\frac{1}{N} \sum_{i=1}^{N} S_{i} I_{[10(k-1), 10 k)}\left(H_{i}\right), 1 \leq k \leq 36, \\
B_{37} & =\frac{1}{N} \sum_{i=1}^{N}\left(1-S_{i}\right),
\end{aligned}
$$

where $I$ is the indicator function, and $H_{i}$ and $S_{i}$ are the first two components in the HSV color space of the $i$-th pixel (in the ranges $[0,360)$ and $[0,1]$, respectively).

The third feature is based on a histogram of Local Binary Patterns (LBP), which is a highly discriminative texture descriptor [15]. Its robustness comes from the invariance with respect to rotations and contrast. Moreover, the descriptor is very compact, and can be computed very quickly. Briefly, the LBP descriptor is defined as a histogram of the local patterns surrounding each pixel of the region. These patterns are computed by thresholding the intensity of the neighbors of each pixel with the intensity of the pixel itself (see Figure 2). The resulting binary code is then rotated to achieve rotation invariance. More in detail, given the neighbor size $P$ and a radius $R$, for each pixel the numerical code $L B P_{P, R}$ is computed as follows:

$$
L B P_{P, R}=\sum_{p=0}^{P-1} s\left(g_{p}-g_{c}\right) 2^{p},
$$

where $g_{c}$ is the gray level of the current pixel, $g_{0}, \ldots, g_{P-1}$ are the gray levels of its neighbors, and $s$ is defined as $s(x)=1$ if $x \geq 0, s(x)=0$ otherwise. The 


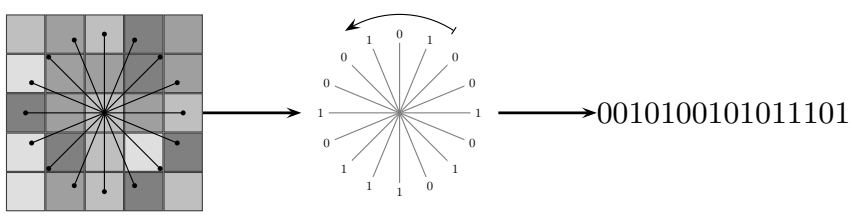

Fig. 2. The first steps of the Local Binary Patter extraction. For each pixel, a circular neighborhood is considered. Each neighbor is thresholded by the intensity of the central pixel determining a binary response. The pattern is formed by concatenating the resulting bits.

$P$ neighbors considered lie on a circular neighbor, of radius $R$, of the current pixel: the gray value $g_{p}$ is obtained by interpolating the intensity image at a displacement $(R \cos (2 \pi p / P)), R \sin (2 \pi p / P))$.

To achieve rotation invariance, the following pattern is defined:

$$
L B P_{P, R}^{r i}=\min _{i=0 \ldots, P-1}\left\{R O R\left(L B P_{P, R}, i\right)\right\}
$$

where $\operatorname{ROR}(x, i)$ performs $i$ times a circular bit-wise right shift on the $P$-bits number $x$. In terms of image pixels this corresponds to rotating the neighbor set clockwise so many times that the longest sequence of zeros is placed in front of the code.

In practice, not all patterns are equally significant and only a selection of all the possible $2^{P}$ patterns are individually accounted in the final descriptor. Usually, only patterns describing a somewhat regular neighbor are considered. These patterns are called "uniform" and are defined as those patterns for which there are at most two transitions (bitwise $0 / 1$ changes) between adjacent bits in the code. All non-uniform patterns are considered together in the final descriptor. Since there are $P+1$ uniform patterns $\left(0 \ldots 0000_{2}, 0 \ldots 0001_{2}, 0 \ldots 0011_{2}\right.$, $\left.0 \ldots 0111_{2}, \ldots, 1 \ldots 1111_{2}\right)$ the code $L B P_{P, R}^{r i u}$ assumes $P+2$ values: one for each uniform pattern, and one for all non-uniform patterns.

As texture descriptor, we used a histogram of 18 bins which counts the occurrences of the $L B P_{16,2}^{\text {riu }}$ codes.

The three features are concatenated and fed to a multi-class Support Vector Machine (SVM) [5]. We use the "one-against-one" approach in which $N_{c}\left(N_{c}-\right.$ 1)/2 (where $N_{c}=7$ is the number of classes considered: sky, vegetation, snow, water, ground, street, and sand) binary classifiers are trained to discriminate data from two different classes. Prediction is based on a majority vote among all the trained classifiers (with a random choice in case of tie). Each binary classifier is a SVM with Gaussian RBF kernel. Therefore, before training the kernel scale $\sigma$ and the penalization coefficient $C$ must be set. To do so, for each binary classifier we uniformly sampled the $\sigma C$ plane and we selected the parameter pair with the highest accuracy estimated by five-fold cross-validation.

The introduction of a rejection option has several advantages: i) regions that are to be classified are not compulsorily assigned to one of the designated classes; ii) ambiguous regions, that is, regions that may be labeled differently by different 
observers, are likely to be rejected; and iii) classification accuracy for non ambiguous regions is improved. The rejection option is based on estimates of posterior probabilities that the input region belongs to the classes considered, given the value of the features. To estimate the probabilities we used the method proposed by $\mathrm{Wu}$ et al. 22. A region is rejected if the maximum of these probabilities is less than $\alpha$ times larger than the second largest probability (where $\alpha$ is a tunable parameter). Otherwise the region is assigned to the class having the largest posterior probability.

\section{Experimental Results}

To train and to evaluate our method we used the annotated images provided by the LabelMe project [17. The dataset is dynamically growing and, as of October 31 2010, includes 62,197 annotated images. Images are annotated online by users who manually segment the images into polygonal regions which are labeled with freely chosen keywords (one keyword per region).

For this work we downloaded all the available annotated images and we looked for keywords matching the seven classes considered. Different keywords may be used to indicate objects matching the classes: Table 1 reports the keyword we selected for each class, together with the resulting number of annotated images containing regions of that class. Note that the images usually contain regions of different classes: a total of 12,755 images has been used during the experiments. These images have been resampled in such a way that the longest side is exactly 1024 pixels long.

To train the classifier, we segmented the images using the approach described in Section 3.1. Each segmented region has been compared with the manual annotation. Only regions with an area exceeding the 10,000 pixels are considered. Moreover, if more than $95 \%$ of pixels of a segmented region belong to regions

Table 1. The keywords we selected for each class, the number of annotated regions, and the number of annotated images containing those regions

\begin{tabular}{llcc}
\hline Class & Keywords & Number of regions Number of images \\
\hline Ground & ground, soil, desert ground, rock & 2,982 & 1,510 \\
Sand & sand, sand beach, beach, desert, & 1,387 & 523 \\
& shore, dune & & \\
Sky & sky, cloud, clouds & 14,446 & 7,398 \\
Snow & snow, snow covered ground & 399 & 222 \\
Street & street, asphalt, pavement, high- & 4,635 & 4,862 \\
& way, road & & 7,281 \\
Vegetation & tree, trees, palm tree, pine tree, & 9,870 & \\
& vegetation, grass, forest, bush, & & 1,051 \\
& bushes, field \\
Water & water, river, sea, sea water, lake, & 2,330 & \\
& river water & & \\
\hline
\end{tabular}


annotated with one of the keywords considered, then that region is further processed as a region of the corresponding class; otherwise the region is discarded. The final number of regions considered is 40,396 as detailed in Table 1, Figure 4 and Figure 5 show a sample of the regions selected for the classes sky and vegetation, respectively. Note that some of the selected regions have been mislabeled. Often this happens when the manual segmentation is too coarse. For instance, sometimes the sky region has been segmented together with trees and buildings, and the automatic segmentation process isolates these objects from the sky. This fact probably causes some degradation in the classification performance. In this work we decided to rely on the annotation provided with the LabelMe dataset. In future works, we plan to manually refine the regions obtained.

The feature vectors extracted from the selected regions has been divided into a training and a test set. For each class 2,000 vectors have been randomly selected for training, with the exception of the sand and snow classes for which all the available feature vectors have been selected (1,387 and 399, respectively). After the model selection and the training procedure, the remaining feature vectors

Table 2. Confusion matrix of classification results obtained on the test set (reported in percentage)

\begin{tabular}{lccccccc}
\hline & \multicolumn{6}{c}{ Predicted class } \\
\cline { 2 - 8 } Correct class & Ground Sand & Sky & Snow & Street & Vegetation & Water \\
\hline Ground & $\mathbf{7 0 . 0 6}$ & 6.92 & 3.05 & 1.43 & 5.80 & 8.76 & 3.97 \\
Sky & 2.03 & 1.15 & $\mathbf{8 6 . 5 3}$ & 0.76 & 0.92 & 5.54 & 3.06 \\
Street & 4.78 & 3.76 & 1.18 & 0.61 & $\mathbf{8 3 . 9 8}$ & 0.99 & 4.71 \\
Vegetation & 10.42 & 1.54 & 2.95 & 0.23 & 0.71 & $\mathbf{8 2 . 0 3}$ & 2.12 \\
Water & 6.97 & 2.42 & 1.52 & 0.61 & 4.85 & 4.24 & $\mathbf{7 9 . 3 9}$ \\
\hline
\end{tabular}

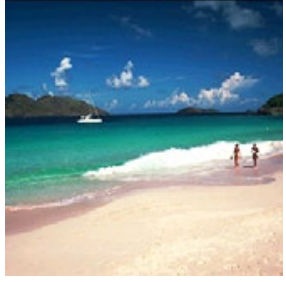

(a) Input image

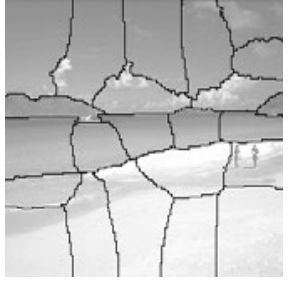

(b) Segmentation

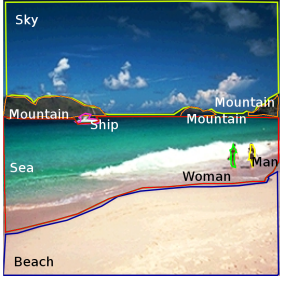

(c) LabelMe annotation

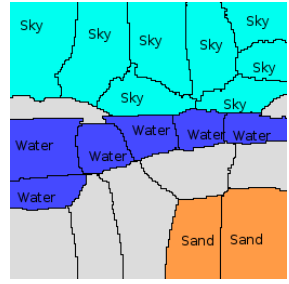

(d) Final labeling

Fig. 3. Example of the procedure used to determine the ground truth for the experimentation. The input image is segmented using the method described in Section 3.1 the resulting regions are compared with the LabelMe annotation to determine the final labeling. Note that some regions are left unlabeled, for instance, because their keywords are not relevant (mountain, ship, woman, man), or because they are ambiguous (the shoreline). 


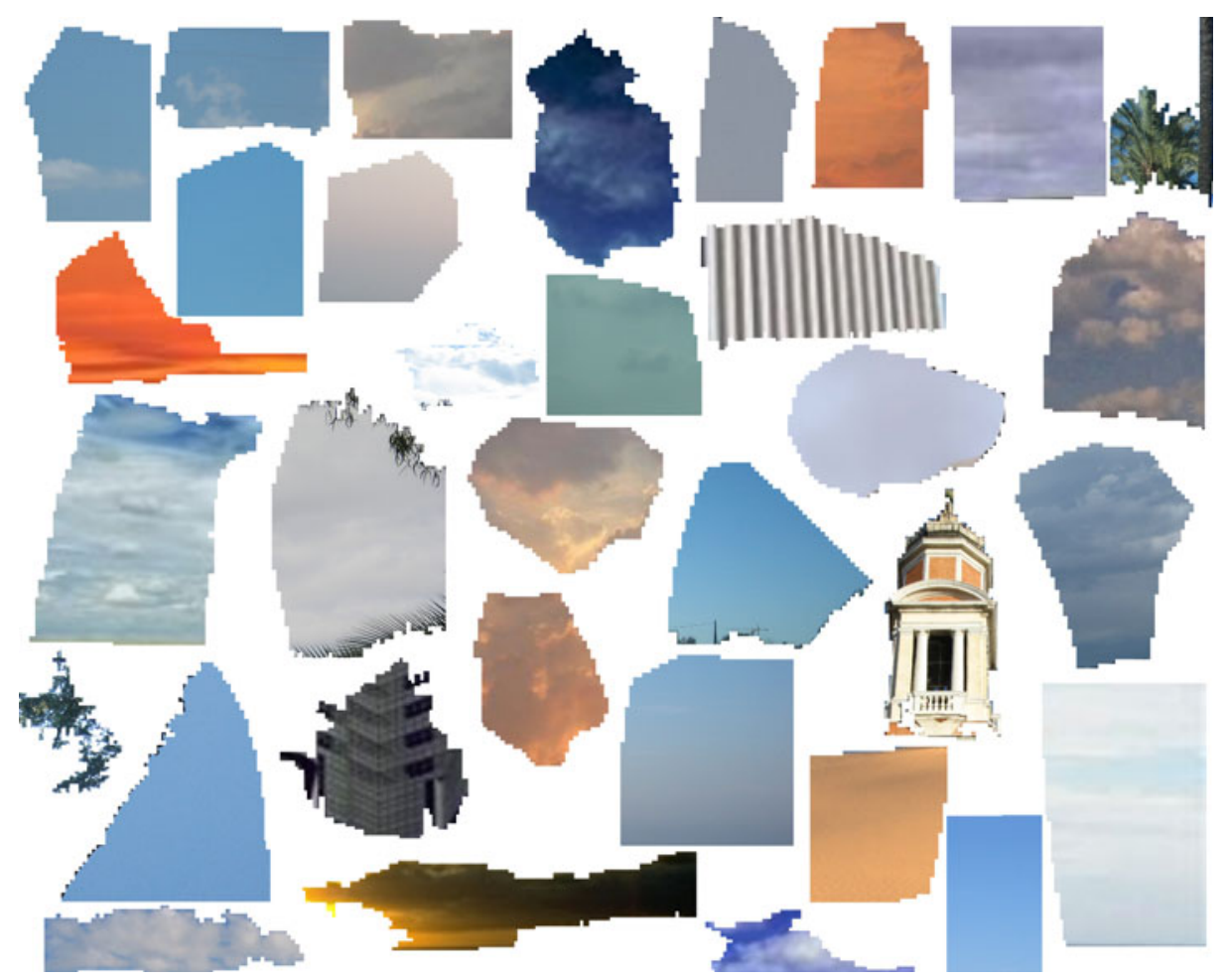

Fig. 4. A sample of the regions considered for the "sky" class

have been used to evaluate the classifier. The estimated accuracies are reported, as a confusion matrix, in Table 2. Note that, due to the lack of feature vectors, the test set of the sand and snow classes are empty. Therefore, we excluded these classes from the performance evaluation and from any further analysis.

The overall classification accuracy on ground, sky, street, vegetation, and water is $80.4 \%$. Most of the errors concern the ground class which is often misclassified as sand $(6.92 \%)$ street $(5.80 \%)$, or vegetation $(8.76 \%)$. Moreover, vegetation and water classes are often classified as ground (10.42\% and $6.97 \%$, respectively). Other common errors are sky regions classified as vegetation (5.54\%) and water regions classified as street $(4.85 \%)$. It should be noted that we completely trusted the LabelMe annotators and that part of the misclassifications could have been caused by annotation errors or inconsistencies.

The rejection option can be used to further improve the accuracy of the classifier. As described before, a region is rejected if the ratio between the two largest estimated posterior probabilities is below a threshold. Figure 6 illustrates that the higher the threshold, the better the classification accuracy is (measured as the mean accuracy of the five classes for which there is a proper test set). That is, rejecting more regions improves classification results on non-rejected regions. 


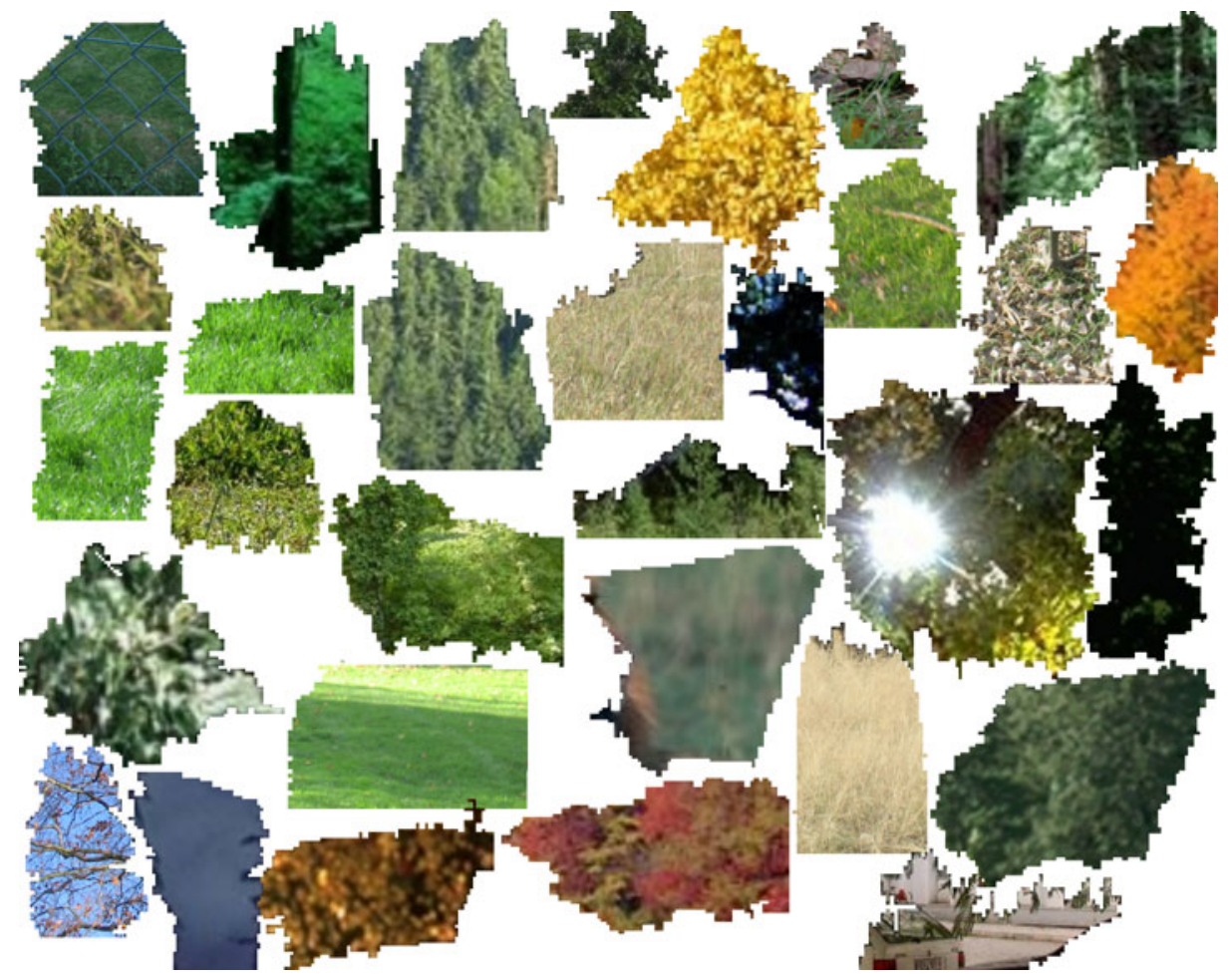

Fig. 5. A sample of the regions considered for the "vegetation" class

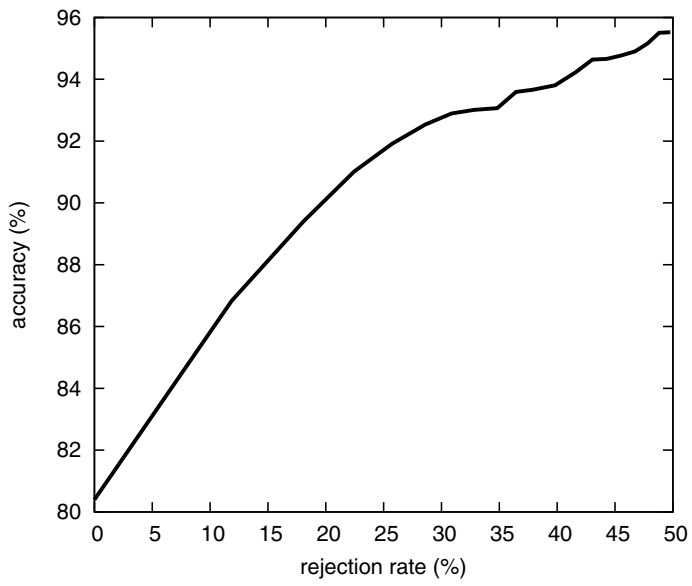

Fig. 6. Trade-off between the amount of rejected regions and the classification accuracy on non-rejected regions 


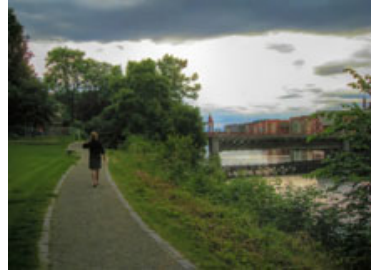

(a) Input image

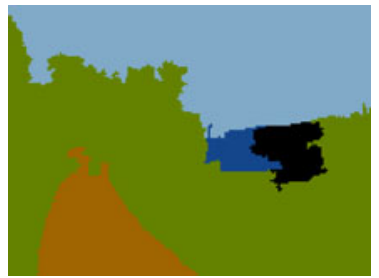

(d) $\alpha=1.5$

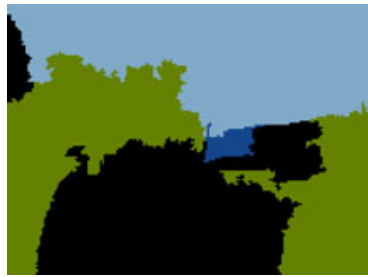

(g) $\alpha=6$

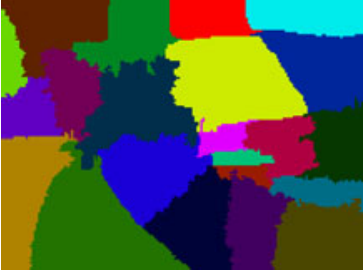

(b) Segmentation

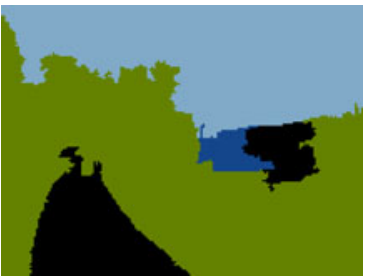

(e) $\alpha=2$

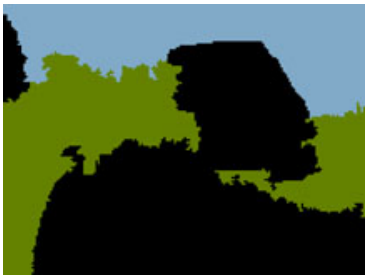

(h) $\alpha=9$

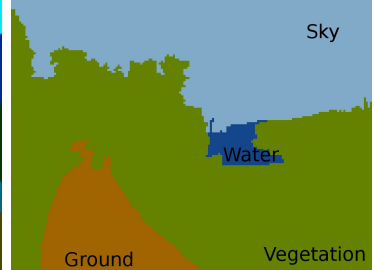

(c) No rejection

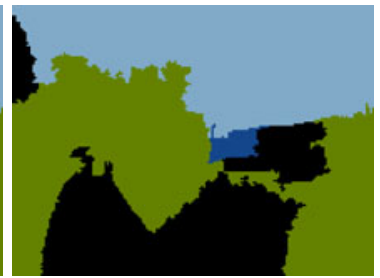

(f) $\alpha=3$

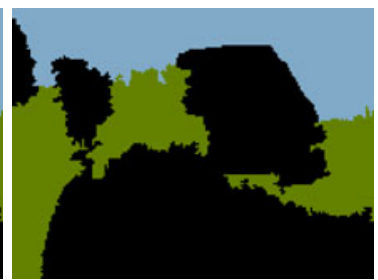

(i) $\alpha=12$

Fig. 7. Examples of the application of the rejection rule: (a) input image; (b) segmentation; (c) annotation without rejection; $(\mathrm{d}-\mathrm{i})$ rejection rule applied with different values of the threshold $\alpha$. Black areas correspond to rejected regions.

For instance, if about $20 \%$ of regions are rejection (corresponding to a threshold value of 4 ) the average accuracy exceeds $91 \%$. The rejection option is also very useful when the method is applied to whole images. In this case, in fact, several regions do not match any of the classes considered. Figure 7 an example of the application of the rejection rule, for different values of the rejection threshold.

Figure 8 reports some examples of application of the annotation strategy, with the rejection threshold $\alpha$ set to the value of 2 . As it can be seen, the final annotation is able to capture the most prominent elements composing the pictures. A common source of errors is the segmentation stage which sometimes fails to isolate the objects in the images. Another cause of error is due to the rejection option, which has not been always able to discard those regions corresponding to non relevant concepts. However, this could be fixed by increasing the rejection threshold, at the expenses of a larger number of rejected regions. Classification of water requires a special remark: very often regions of this class present specular reflections which 


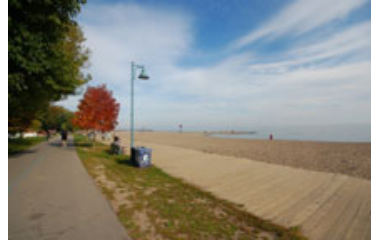

(a) Input image

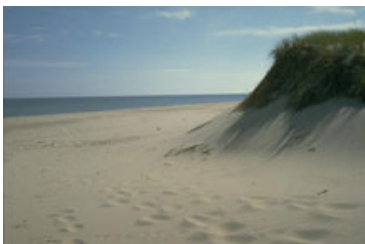

(d) Input image

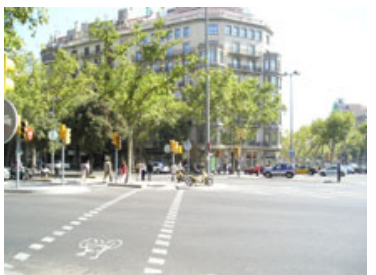

(g) Input image

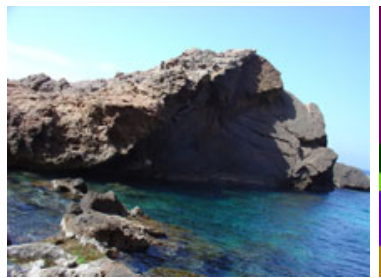

(j) Input image

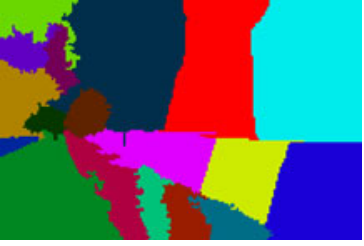

(b) Segmentation

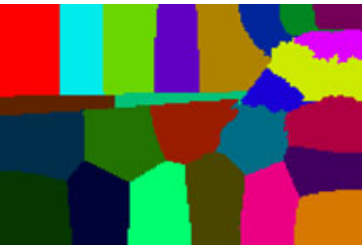

(e) Segmentation

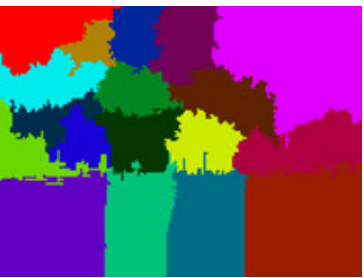

(h) Segmentation

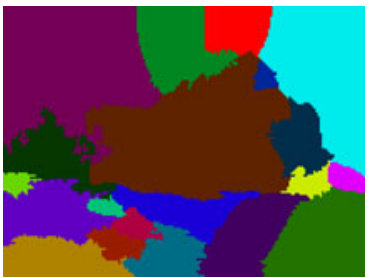

(k) Segmentation

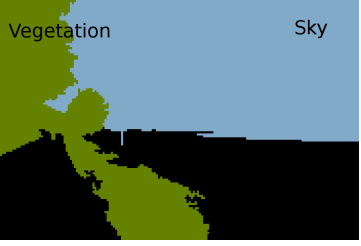

(c) Annotation

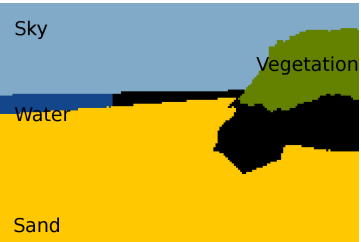

(f) Annotation

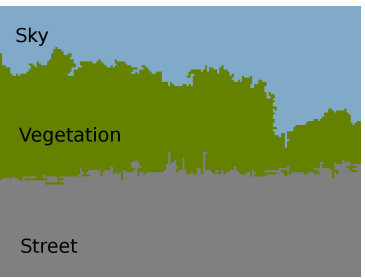

(i) Annotation

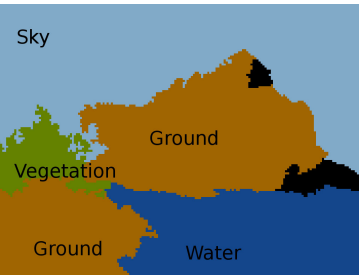

(l) Annotation

Fig. 8. Examples of annotation. For each input image (a, d, g, j) are shown their segmentation (b, e, h, k), and their final annotation (c, f, i, l). Rejected regions have been painted in black (for these examples we set $\alpha=2$ ).

may deceive the classifier since, in these cases, the features represent the reflected objects, not the water itself.

\section{Conclusions}

We presented a strategy for the automatic annotation of outdoor photographs. Images are over segmented by applying a normalized cut algorithm tuned to 
select regions composed of highly homogeneous pixels. The regions are then independently classified by a multi-class Support Vector Machine into the classes sky, vegetation, snow, water, ground, street, and sand. The classification accuracy obtained on the LabelMe dataset is higher than $80 \%$. A rejection rule may also be applied to improve the accuracy of the classifier.

We plan to exploit the information provided by the proposed strategy to improve computational color algortihms. For instance, we plan to exploit the automatic annotation for the proble of computational color constancy, as described by Gijsenij and Gevers [10].

We believe that automatic image annotation could also be applied to improve content-based image retrieval systems. In fact, the majority of systems in the state of the art are based on low-level features such as color and texture statistics, or bag-of-features descriptors. The detection of semantically meaningful regions could improve the accuracy of these systems, by reducing the semantic gap between the user's intentions and the representations used to index the images. The information provided by our strategy could also be exploited to further classify the content of the images, for instance, by following the approach proposed by Cheng and Wang [2].

\section{References}

1. Boutell, M., Luo, J., Shen, X., Brown, C.: Learning multi-label scene classification. Pattern Recognition 37(9), 1757-1771 (2004)

2. Cheng, H., Wang, R.: Semantic modeling of natural scenes based on contextual Bayesian networks. Pattern Recognition 43(12), 4042-4054 (2010)

3. Ciocca, G., Cusano, C., Gasparini, F., Schettini, R.: Content aware image enhancement. In: Basili, R., Pazienza, M.T. (eds.) AI*IA 2007. LNCS (LNAI), vol. 4733, pp. 686-697. Springer, Heidelberg (2007)

4. Cooper, T.: Color segmentation as an aid to white balancing for digital still cameras, 4300, 164-171 (2000)

5. Cortes, C., Vapnik, V.: Support-vector networks. Machine Learning 20, 273-297 (1995)

6. Cusano, C., Ciocca, G., Schettini, R.: Image annotation using SVM. In: Proc. of Internet Imaging V. SPIE, vol. 5304, pp. 330-338 (2004)

7. Cusano, C., Gasparini, F., Schettini, R.: Image annotation for adaptive enhancement of uncalibrated color images. In: Bres, S., Laurini, R. (eds.) VISUAL 2005. LNCS, vol. 3736, pp. 216-225. Springer, Heidelberg (2006)

8. Fredembach, C., Estrada, F., Süsstrunk, S.: Memory colour segmentation and classification using class-specific eigenregions. Journal of the Society for Information Display 17(11), 921-931 (2009)

9. Gasparini, F., Schettini, R.: Color balancing of digital photos using simple image statistics. Pattern Recognition 37(6), 1201-1217 (2004)

10. Gijsenij, A., Gevers, T.: Color constancy using image regions. In: IEEE International Conference on Image Processing, vol. 3, pp. 501-504 (2007)

11. Guillaumin, M., Mensink, T., Verbeek, J., Schmid, C.: Tagprop: Discriminative metric learning in nearest neighbor models for image auto-annotation. In: IEEE 12th International Conference on Computer Vision, pp. 309-316 (2010) 
12. Jeon, J., Lavrenko, V., Manmatha, R.: Automatic image annotation and retrieval using cross-media relevance models. In: Proceedings of the 26th Annual International ACM SIGIR Conference on Research and Development in Informaion Retrieval, pp. 119-126 (2003)

13. Malik, J., Belongie, S., Leung, T., Shi, J.: Contour and texture analysis for image segmentation. International Journal of Computer Vision 43, 7-27 (2001)

14. Millet, C., Bloch, I., Hede, P., Moellic, P.: Using relative spatial relationships to improve individual region recognition. In: European Workshop on the Integration of Knowledge, Semantics and Digital Media Technologies, EWIMT, vol. 5, pp. 119-126 (2005)

15. Ojala, T., Pietikäainen, M., Mäaenpää, T.: Multiresolution gray-scale and rotation invariant texture classification with local binary patterns. IEEE Transactions on Pattern Analysis and Machine Intelligence 24(7), 971-987 (2002)

16. Rui, X., Li, M., Li, Z., Ma, W., Yu, N.: Bipartite graph reinforcement model for web image annotation. In: Proceedings of the 15th International Conference on Multimedia, pp. 585-594 (2007)

17. Russell, B., Torralba, A., Murphy, K., Freeman, W.: LabelMe: a database and webbased tool for image annotation. International Journal of Computer Vision 77(1), 157-173 (2008)

18. Shi, J., Malik, J.: Normalized cuts and image segmentation. IEEE Transactions on Pattern Analysis and Machine Intelligence 22(8), 888-905 (2000)

19. Tsai, C., Hung, C.: Automatically annotating images with keywords: A review of image annotation systems. Recent Patents on Computer Science 1(1), 55-68 (2008)

20. Wang, C., Jing, F., Zhang, L., Zhang, H.: Content-based image annotation refinement. In: IEEE Conference on Computer Vision and Pattern Recognition, pp. 1-8 (2007)

21. Van de Weijer, J., Gevers, T., Bagdanov, A.: Boosting color saliency in image feature detection. IEEE Transactions on Pattern Analysis and Machine Intelligence 28(1), 150-156 (2006)

22. Wu, T., Lin, C., Weng, R.: Probability estimates for multi-class classification by pairwise coupling. The Journal of Machine Learning Research 5, 975-1005 (2004)

23. Yuan, J., Li, J., Zhang, B.: Exploiting spatial context constraints for automatic image region annotation. In: Proceedings of the 15th International Conference on Multimedia, pp. 595-604 (2007) 